University of Vermont

UVM ScholarWorks

$2-1-2010$

\title{
Sensitivity of incipient particle motion to fluid flow penetration depth within a packed bed
}

\author{
Benjamin A. Yergey \\ Bucknell University \\ Maria Laura Beninati \\ Bucknell University \\ Jeffrey S. Marshall \\ University of Vermont
}

Follow this and additional works at: https://scholarworks.uvm.edu/cemsfac

\section{Recommended Citation}

Yergey BA, BENINATI ML, Marshall JS. Sensitivity of incipient particle motion to fluid flow penetration depth within a packed bed. Sedimentology. 2010 Feb;57(2):418-28.

This Article is brought to you for free and open access by the College of Engineering and Mathematical Sciences at UVM ScholarWorks. It has been accepted for inclusion in College of Engineering and Mathematical Sciences Faculty Publications by an authorized administrator of UVM ScholarWorks. For more information, please contact scholarworks@uvm.edu. 


\title{
Sensitivity of incipient particle motion to fluid flow penetration depth within a packed bed
}

\author{
BENJAMIN A. YERGEY*, MARIA-LAURA BENINATI* and JEFFREY S. MARSHALL $\dagger$ \\ * Mechanical Engineering Department, Bucknell University, 701 Moore Avenue, Lewisburg, PA 17837, \\ USA (E-mail: mlb041@bucknell.edu) \\ $\dagger$ School of Engineering, University of Vermont, Burlington, VT 05405, USA
}

Associate Editor - Subhasish Dey

\begin{abstract}
A discrete element method is applied to a three-dimensional analysis related to sediment entrainment on a micro-scale. Sediment entrainment is the process by which a fluid medium accelerates particles from rest and advects them upward until they are either transported as bedload or suspended by the flow. Modelling of the entrainment process is a critically important aspect for studies of erosion, pollutant resuspension and transport, and formation of bedforms in environmental flows. Previous discrete element method studies of sediment entrainment have assumed the flow within the particle bed to be negligible and have only allowed for the motion of the topmost particles. At the same time, micro-scale experimental studies indicate that there is a small slip of the fluid flow at the top of the bed, indicating the presence of nonvanishing fluid velocity within the topmost bed layers. The current study demonstrates that the onset of particle incipient motion, which immediately precedes particle entrainment, is highly sensitive to this small fluid flow within the topmost bed layers. Using an exponential decay profile for the inner-bed fluid flow, the discrete element method calculations are repeated with different fluid penetration depths within the bed for several small particle Reynolds numbers. For cases with slip velocity corresponding to that observed in previous experiments with natural sediment, the predicted particle velocity is found to be a few percent of the fluid velocity at the top of the viscous wall layer, which is a reasonable range of velocities for observation of incipient particle motion. This method for prescribing the fluid flow within the particle bed allows for the current discrete element method to be extended in future studies to the analysis of sediment entrainment under the influence of events such as turbulent bursting. Additionally, predictions for the slip velocities and fluid flow profile within the bed suggest the need for further experimental studies to provide the data necessary for additional improvement of the discrete element method models.
\end{abstract}

Keywords Discrete element method, incipient motion, particle bed, penetration depth, porous media, sediment entrainment, slip velocity.

\section{INTRODUCTION}

Prediction of sediment particle incipient motion in boundary-layer flows, which immediately precedes sediment entrainment, has been a focus of research for over seven decades. The classic research on this subject was performed by Shields (1936) for turbulent boundary layer flows over flat beds by balancing the drag on a particle with the gravitational forces resisting motion. By 
extrapolating experimental results for sediment discharge versus bed shear stress to a state of zero sediment discharge, Shields (1936) obtained the well-known relationship for the critical fluid shear stress at which the onset of particle motion occurs. However, as noted by Shields (1936) and numerous subsequent researchers (e.g. Vanoni, 1975), the process of incipient sediment motion is statistical in nature and no truly critical condition exists at which the sediment starts moving all at once. Rather, there is an interval of the bed shear stress over which the fraction of sediment in motion increases rapidly, leading to a state in which the particles in the top layer are nearly all in motion.

Many subsequent investigators report data for incipient particle motion under different conditions for both laminar and turbulent flows (e.g. White, 1940; Egiazaroff, 1950; Tison, 1953; Shvidchenko \& Pender, 2000; Pender et al., 2007). Ling (1995) proposed separate theoretical thresholds for incipient rolling and lifting motions of a particle. For uniform spherical particles, Ling considered the fluid velocity around a spherical particle to be purely streamwise and to vary linearly with height (an assumption used for the viscous sub-region in turbulent boundary layers). The thresholds developed determine the critical fluid shear stress required for incipient motion of particles either in a rolling motion over stationary particles or a lifting motion from a bed of stationary particles.

There has recently been renewed interest in modelling of incipient particle motion. In part, this interest derives from the recent use of largeeddy simulation (LES) methods for sediment transport turbulent flows (e.g. Zedler \& Street, 2001; Chang \& Scotti, 2003) which require improved time-accurate models for sediment entrainment into the flow. At the same time, recent findings suggest that the classical models of incipient motion may have left out some important aspects of the problem. For instance, Foster et al. (2006) demonstrate that particle incipient motion can occur due to exposure of particles to a pressure gradient, such as might be caused by a passing free-surface wave or vortex; this is an entirely distinct mechanism for incipient motion from the shear-stress driven motion described in the classical studies. Hammond et al. (1984) and Fenton \& Abbott (1977) note the deviation from the Shields criterion observed for particles with different relative protrusion into the flow, such as might occur for beds composed of particles of different sizes or loosely packed particles. Zanke (2003) reinterpreted a variety of incipient motion data to account for the effect of fluctuations in the turbulent boundarylayer flow. A recent review of research on incipient particle motion is given by Dey \& Papanicolaou (2008).

A number of studies have noted that the fluid velocity field just above the particulate bed exhibits a non-zero slip velocity, indicating the presence of shear-driven fluid flow within the topmost part of the particle bed (Brinkman, 1947; Beavers \& Joseph, 1967; Taylor, 1971; Larson \& Higdon, 1986, 1987; Sahraoui \& Kaviany, 1992; James \& Davis, 2001; Pokrajac et al., 2007). Beavers \& Joseph (1967) and Taylor (1971) argue that the slip velocity is proportional to the shear stress above the bed. James \& Davis (2001) and Larson \& Higdon $(1986,1987)$ examine the micro-scale flow and resulting shear-driven velocity fields for porous beds composed of arrays of rods. Sangani \& Behl (1989) compare micro-scale analytical solutions of the Stokes equations with the continuum approximations of Brinkman (1947) and Acrivos \& Chang (1986) and find that the continuum approximations remain reasonably accurate even when the length-scale associated with the velocity decay within the bed is of the order of the particle diameter. Based on experiments with flow in a flume over flat sand beds with different grain sizes, Gyr \& Schmid (1997) present fluid velocity profiles that exhibit dimensionless slip velocities $u_{+} \equiv u / u_{*}$ at the bed surface ranging from 0 (for a hydraulically smooth bed) to nearly 8 for values of the dimensionless bed roughness height $k_{+} \equiv k u * / v<2 \cdot 8$, where $u_{*} \equiv\left(\tau_{w} / \rho\right)^{1 / 2}$ is the friction velocity and $v$ is the fluid kinematic viscosity. The relationship between fluid slip at the sediment-water interface and interfacial diffusion coefficients and deposition of small particles is examined by Fries (2007) and Fries \& Trowbridge (2003). The increase in slip velocity with boundary suction in a porous medium is explored by Chen \& Chiew (2004).

Detailed discussions of the forces acting on particles near the sediment-water interface and the role of these forces in various models of particle incipient motion are given by Phillips (1980) and more recently by Niño et al. (2003). Implementation of individual particle force models for flow over a packed bed has been presented by Drake \& Calantoni (2001), McEwan \& Heald (2001) and Schmeeckle \& Nelson (2003) using so-called 'discrete particle' or 'discrete element methods' (DEM). McEwan \& Heald (2001) calculate the required destabilizing force for particles 
to begin moving, considering each topmost particle to be in contact with exactly three other particles. Schmeeckle \& Nelson (2003) assume the vertical momentum transport within the bed to be dominated by the bed drag and for a particle to be considered for motion it must be at the surface of the bed. Drake \& Calantoni (2001) examine bedload transport of particles under wave-induced fluid oscillations of different forms. All these DEM studies neglect the effects of fluid slip and fluid motion within the bed.

The current paper incorporates fluid slip velocity and flow within the topmost part of the sediment bed into a DEM simulation in order to examine the sensitivity of the computational results for particle incipient motion to the form of the velocity field within the bed. The internalbed fluid velocity profile is incorporated into a DEM model which allows for motion of all sediment particles. The sensitivity of the DEM predictions to variation in penetration depth is examined, and an estimate for bed penetration depth is developed using previous data for incipient particle motion in comparison to the computational results.

The theoretical model for the fluid flow profile within the bed is discussed in the Theory for velocity profile within the bed section. In the Computational method section, a summary of the computational model is provided, which is based on the recent DEM model of Marshall (2009). The set-up and various parameters used for the numerical computations are discussed in the Problem setup section. Results of sensitivity tests for dependence of particle incipient model on fluid penetration depth are presented in the Computational results section. Conclusions are given in the Discussion and Conclusions section.

\section{THEORY FOR VELOCITY PROFILE WITHIN THE BED}

The fluid-particle interfacial force introduces a body force $\mathbf{f}$ in the momentum conservation equation, to yield the mass conservation and Navier-Stokes equations for fluid flow of the form (Zhao et al., 2008; Marshall, 2009):

$$
\begin{gathered}
\frac{\partial}{\partial t}\left(\varepsilon \rho_{\mathrm{f}}\right)+\nabla \cdot\left(\varepsilon \rho_{\mathrm{f}} \mathbf{u}\right)=0 \\
\rho_{\mathrm{f}} \varepsilon\left[\frac{\partial \mathbf{u}}{\partial t}+(\mathbf{u} \cdot \nabla) \mathbf{u}\right]=-\varepsilon \nabla p+\nabla \cdot(2 \varepsilon \mu \mathbf{D})-\mathbf{f}
\end{gathered}
$$

where $\mathbf{D}$ is the rate of deformation tensor and $\mathbf{u}$ is the fluid velocity field of a spatially averaged fluid flow within the local pore space, $\rho_{\mathrm{f}}$ and $\mu$ are the fluid density and viscosity, respectively, and the void fraction $\varepsilon$ is expressed in terms of the particle concentration field $\phi$ as $\varepsilon=1-\phi$. Within the particle bed, the common assumption is made that the flow is sufficiently slow that inertial terms are negligible (Bear, 1988). It is also assumed that the length-scale of the velocity field $\mathbf{u}$ is much smaller in the bed-normal $y$-direction than in the $x$-direction, so that the usual boundary layer approximation applies. If the particle concentration is uniform within the bed, Eq. 2 reduces to:

$$
\nabla p=\mu \frac{\mathrm{d}^{2} \mathbf{u}}{\mathrm{d} y^{2}}-\frac{1}{\varepsilon} \mathbf{f}
$$

The interfacial force for stationary particles is given by (Bear, 1988):

$$
\mathbf{f}=(\mu \varepsilon / B) \mathbf{u}
$$

where $B$ is the average conductance of the porous medium. Substituting Eq. 4 into Eq. 3 yields:

$$
\nabla p=\mu \frac{\mathrm{d}^{2} \mathbf{u}}{\mathrm{d} y^{2}}-\frac{\mu}{B} \mathbf{u}
$$

Equation 5 is the same as the expression derived by Brinkman (1947) for flow through a "particle swarm'. For a pressure-driven flow the shear term in this equation is negligible and Eq. 5 reduces to the well-known Darcy's law (Darcy, 1856) relating pressure gradient and velocity of flow through a porous medium.

Integrating Eq. 5 in $y$ with zero pressure gradient yields an exponentially decaying velocity profile of the form:

$$
u(y)=u_{0} \exp (y / P), \quad y<0
$$

where $u_{0}$ is the slip velocity at the bed surface $(y=0)$ and $P \equiv \sqrt{B}$ is the characteristic lengthscale of exponential velocity decay within the bed, which is referred to here to as the 'penetration depth'. The bed surface $(y=0)$ is defined as the average value of the topmost point on the set of uppermost particles within the bed. Matching Eq. 6 to a linear velocity profile $u(y)=S y+u_{0}$ above the bed, where $S$ is the shear rate, and requiring that both the velocity and the velocity gradient are continuous at $y=0$ yields an expression for slip velocity in terms of shear rate and penetration depth as: 


$$
u_{0}=S P
$$

Dimensionless variables are introduced using the wall (or viscous) scaling for a turbulent boundary layer, so as to better enable comparison with experimental data for turbulent boundary layers, as:

$$
u_{+}=\frac{u}{u_{*}}, y_{+}=\frac{y u_{*}}{v}, P_{+}=\frac{P u_{*}}{v}, \quad S_{+}=\frac{S v}{u_{*}^{2}}, \quad u_{0,+}=\frac{u_{0}}{u_{*}}, t_{+}=\frac{t u_{*}^{2}}{v}
$$

Using the fact that $\tau_{\mathrm{w}}=\mu \mathrm{S}$ and the definition of the friction velocity $u_{*} \equiv\left(\tau_{\mathrm{w}} / \rho_{\mathrm{f}}\right)^{1 / 2}$ gives $S_{+}=1$, so that Eq. (7) becomes $u_{0_{+}}=P_{+}$and the dimensionless velocity profile can be expressed in terms of the dimensionless fluid penetration depth $P_{+}$as:

$$
u_{+}= \begin{cases}y_{+}+P_{+} & \text {for } y_{+} \geq 0 \\ P_{+} \exp \left(y_{+} / P_{+}\right) & \text {for } y_{+}<0\end{cases}
$$

A schematic showing the form of the velocity profile is given in Fig. 1. The predicted exponential decay of the velocity profile within the bed is consistent with the Brinkman (1947) prediction.

\section{COMPUTATIONAL METHOD}

The particle motion is computed using a discrete element method (DEM) developed by Marshall (2006, 2007, 2009). The computational method uses a multiple time step algorithm, in which the fluid time step $\Delta t=O(L / U)$, the particle time step $\Delta t_{\mathrm{p}}=O(d / U)$ and the collision time step

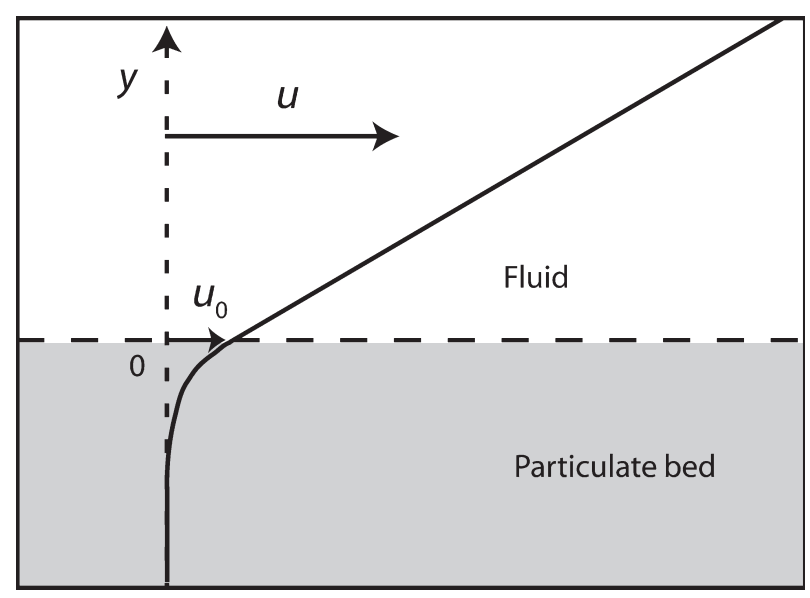

Fig. 1. Fluid velocity profile for flow through and above a porous medium, where $u_{0}$ is the slip-velocity and $u(y)$ is the fluid velocity as a function of height. focusing on the terms of most importance for prediction of particle incipient motion.

The method follows the motion of individual particles by solution of the particle momentum and angular momentum equations using the action of fluid-induced and collision forces and torques. The dominant fluid force is the drag force, approximated by a modified form of the Stokes drag law:

$$
\mathbf{F}_{\mathrm{d}}=-3 \pi d \mu(\mathbf{v}-\mathbf{u}) f
$$
velocities and $f$ is a friction factor that accounts for the effect of local particle crowding, which takes on the value $f=1$ for an isolated sphere. A correlation of Di Felice (1994) is used for $f$ as a function of local particle concentration $\phi$ and particle Reynolds number. The associated fluid torque arises from a difference in rotation rate of the particle and the local fluid region and is given by:

$$
\mathbf{M}_{\mathrm{F}}=-\pi \mu d^{3}\left(\Omega-\frac{1}{2} \boldsymbol{\omega}\right)
$$

where $\Omega$ is the particle rotation rate and $\omega$ is the local fluid vorticity vector.

The lift force acting on a spherical particle at the top of the bed is not well-understood because of the effect of the sharp concentration gradient. For this reason, a number of DEM studies have neglected lift forces (e.g. McEwan \& Heald, 2001; Schmeeckle \& Nelson, 2003). In the current study, the lifting force on the particle is approximated roughly as the sum of the Saffman and Magnus lift expressions. The Saffman lift force (Saffman, 1965, 1968) gives the lift on a particle with small particle Reynolds number, $\operatorname{Re}_{P} \equiv|\mathbf{v}-\mathbf{u}| d / v<O(1)$, rotating at the same rate as the local fluid rotation $(\omega / 2)$ as:

$$
\mathbf{F}_{\ell}=-2 \cdot 18 \chi m \frac{(\mathbf{v}-\mathbf{u}) \times \boldsymbol{\omega}}{\operatorname{Re}_{P}^{1 / 2} \alpha^{1 / 2}}
$$

where $\chi=\rho_{\mathrm{f}} / \rho_{\mathrm{p}}$ is the density ratio, $m$ is the particle mass, and $\alpha \equiv \omega d / 2|\mathbf{v}-\mathbf{u}|$. The Magnus lift force where $\mathbf{v}$ and $\mathbf{u}$ are the particle and local fluid 
gives an additional force on a particle rotating at a rate different from that of the local fluid as:

$$
\mathbf{F}_{m}=-\frac{3}{4} \chi m\left(\frac{1}{2} \boldsymbol{\omega}-\Omega\right) \times(\mathbf{v}-\mathbf{u})
$$

The Magnus force is proportional to the difference in fluid and particle rotation rates, whereas the Saffman force assumes that this difference vanishes. For DEM studies, the particles can experience rapid transient rotations due to collisions, during which time the Magnus term dominates the lift force. Following collisions, the particle rotation rate gradually approaches the local fluid rotation rate due to the effect of the viscous torque $\mathbf{M}_{F}$, at which point the Saffman term dominates the lift force. Additional discussion of models for particle lift at different particle Reynolds numbers is given by Crowe et al. (1998).

Particle collisions are simulated by employing a soft-sphere collision model that treats collisions as a spring and mass damper system, where each collision includes a normal force along the line of collision and frictional resistances for sliding and twisting motions of the particles. No rolling resistance is utilized in the current study. The normal vector $\mathbf{n}$ for a collision between particles $i$ and $j$ is given by:

$$
\mathbf{n}=\left(\mathbf{x}_{j}-\mathbf{x}_{i}\right) /\left|\mathbf{x}_{j}-\mathbf{x}_{i}\right|
$$

where $\mathbf{x}_{i}$ and $\mathbf{x}_{j}$ are the particle centroids. The total collision force and torque on a particle are given by:

$$
\mathbf{F}_{\mathrm{C}}=F_{\mathrm{n}} \mathbf{n}+F_{\mathrm{s}} \mathbf{t}_{\mathrm{S}}, \quad \mathbf{M}_{\mathrm{C}}=r F_{\mathrm{s}}\left(\mathbf{n} \times \mathbf{t}_{\mathrm{S}}\right)+M_{\mathrm{t}} \mathbf{n}
$$

where $F_{\mathrm{n}}$ is the normal force, $F_{\mathrm{s}}$ is the sliding resistance force, $\mathbf{t}_{\mathrm{S}}$ is the unit vector in the direction of sliding, $r$ is the particle radius and $M_{\mathrm{t}}$ is the twisting resistance torque. The normal force is composed of the elastic force $F_{\text {ne }}$ and a dissipative force $F_{\text {nd }}$. The classical Hertz (1882) expression for the elastic normal force is used and the common rate-based expression is used for the dissipative normal force, such that:

$$
F_{\mathrm{ne}}=-k_{N} \delta_{N}=-K \delta_{N}^{3 / 2}, \quad F_{\mathrm{nd}}=\eta_{N} \mathbf{v}_{\mathrm{R}} \cdot \mathbf{n}
$$

Here the particle overlap $\delta_{N}=r_{i}+r_{j}-\left|\mathbf{x}_{i}-\mathbf{x}_{j}\right|$ is written in terms of the radii of the two colliding particles $r_{i}$ and $r_{j}, \mathbf{v}_{R}=\mathbf{v}_{i}-\mathbf{v}_{j}$ is the relative particle velocity and $\eta_{N}$ is the dissipation coefficient. An expression due to Tsuji et al. (1992) is used which gives $\eta_{N}=\bar{\alpha}\left(m k_{N}\right)^{1 / 2}$, where the coefficient $\bar{\alpha}$ can be expressed in terms of the particle restitution coefficient $e$. In general the restitution coefficient varies with particle Stokes number $S t \equiv \rho_{\mathrm{p}} d^{2} U / 18 \mu \mathrm{L}$ (Joseph et al., 2001). For the present study the constant value $e=0.65$ is utilized which is the same value used by Schmeeckle \& Nelson (2003) and is consistent with observed values for natural sediment for the range of Stokes numbers used in the computations (Schmeeckle et al., 2001). The coefficient $K$ is given by $K=k_{N} / \delta_{N}^{1 / 2}=$ (4/3) $E \sqrt{R}$, where $E$ and $R$ are expressed in terms of the particle radii, elastic moduli, and Poisson ratios as:

$$
\frac{1}{R} \equiv \frac{1}{r_{i}}+\frac{1}{r_{j}}, \quad \frac{1}{E} \equiv \frac{1-\sigma_{i}^{2}}{E_{i}}+\frac{1-\sigma_{j}^{2}}{E_{j}}
$$

The spring-dashpot-slider model is used here for the sliding resistance proposed by Cundall \& Strack (1979), in which the tangential sliding force $F_{\mathrm{s}}$ is first absorbed by the spring and dashpot until its magnitude reaches a critical value $F_{\text {crit }}=\mu_{\mathrm{f}}\left|F_{\mathrm{n}}\right|$. An analogous model is employed for twisting resistance torque. The reader is referred to Marshall (2009) for details.

\section{PROBLEM SET-UP}

Computations are performed in a rectangular domain, as shown in Fig. 2. The spanwise and streamwise boundaries are periodic. The bottom plane of the domain is a fixed wall, and the top surface of the domain is a moving wall with velocity equal to the fluid velocity at that height. The particle bed is initialized by distributing particles evenly throughout the computational domain, with small velocities that are randomly perturbed. The particles are allowed to fall to rest at the bottom of the channel, with no fluid flow. A similar initialization procedure was used in the DEM studies by McEwan \& Heald (2001) and Schmeeckle \& Nelson (2003). Upon settling, the bed surface is observed to be flat, so that the maximum particle height in this equilibrium state is identified as the bed surface height for fluid velocity profile calculations. The computations are then restarted with the fluid velocity field turned on. A gradual ramping-up interval of $0<t_{+}<20$ is used to minimize undue disturbance to the particles as the fluid velocity is turned on. The bottom row of particles is fixed to simulate the frictional forces due to additional particles that would exist in an infinite bed. 
Fig. 2. Three-dimensional diagram of the computational domain.

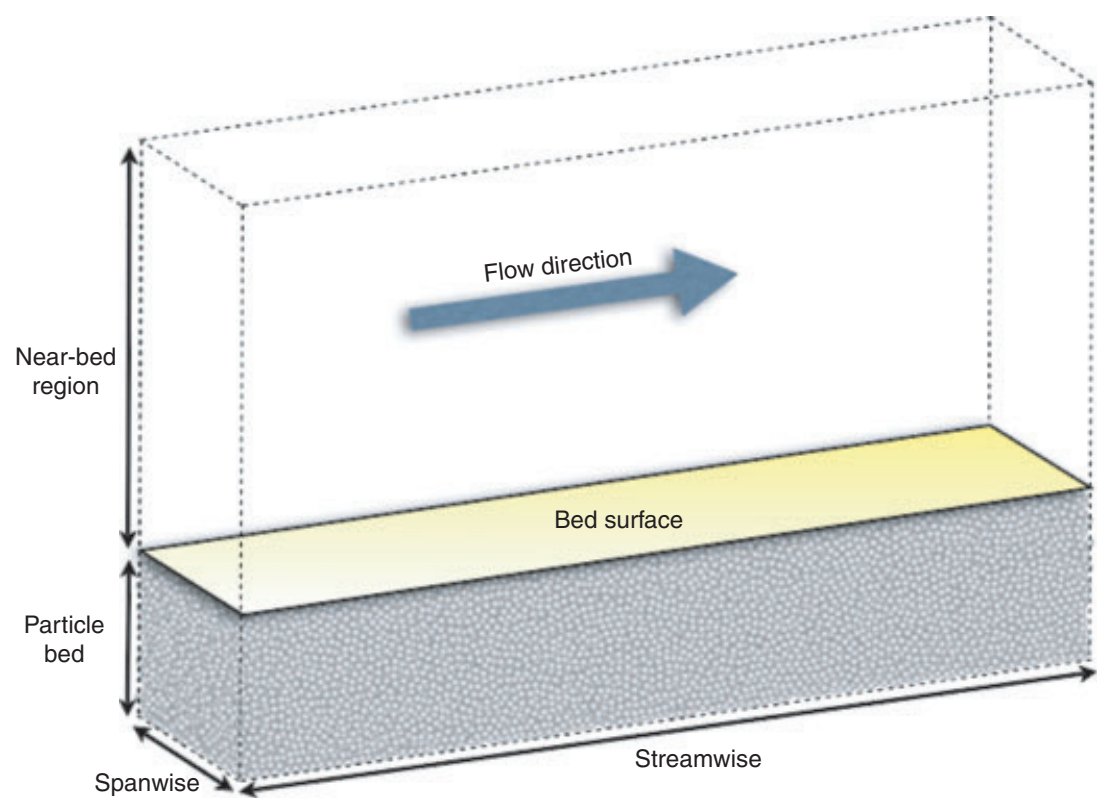

The particle diameters and friction velocities considered are set to correspond with those used in the experiments of Gyr \& Schmid (1997), which were performed for fine sand particles of mean diameter $0.2 \mathrm{~mm}$ and friction velocities ranging from 9 to $15 \mathrm{~mm} \mathrm{~s}^{-1} \cdot d_{+}=d u_{*} / v$ is defined as the particle diameter in wall units, which is equal to the particle Reynolds number scaled with the friction velocity. The computations examine six values of $d_{+}$, ranging from $1 \cdot 3$ to $2 \cdot 7$. The shear rate for all cases is set equal to the critical shear predicted by the Shields (1936) criterion for incipient particle motion, which is usually expressed in terms of a critical value of the dimensionless parameter $\theta_{\text {crit }}=\tau_{\text {crit }} / d g\left(\rho_{p}-\rho_{f}\right)$ as a function of $d_{+}$. For each value of $d_{+}$, runs were performed with six different values of the penetration depth ranging from $P_{+}=0.2$ to 25 , corresponding to between $0 \cdot 1$ to 10 times the particle diameter. The penetration depth values were selected by observations for the range of values for which particles begin moving for the different cases examined. A summary of the parameter values used in the computations is given in Table 1.

The domain size is set to 100 wall units in the vertical and spanwise directions and to 400 wall units in the streamwise direction. The number of particles used varied with particle diameter from a low of about 60000 to a high of 144000 in such a way as to provide a uniform bed depth $H$ for all cases. Using the time step scaling developed by Marshall (2009), the dimensionless fluid, particle and collision time steps for the current computa-
Table 1. Parameter values for the six different flow conditions examined, along with the symbol used for each case in Figs 4 and 5.

\begin{tabular}{llllll}
\hline Case & $d(\mathrm{~m})$ & $u_{*}\left(\mathrm{~m} \mathrm{~s}^{-1}\right)$ & $d_{+}$ & $\theta_{\text {crit }}$ & Symbol \\
\hline 1 & $0 \cdot 00015$ & $0 \cdot 012$ & $2 \cdot 7$ & $0 \cdot 048$ & + \\
2 & $0 \cdot 00015$ & $0 \cdot 0087$ & $1 \cdot 3$ & $0 \cdot 080$ & $\diamond$ \\
3 & $0 \cdot 00015$ & $0 \cdot 0092$ & $1 \cdot 4$ & $0 \cdot 075$ & $\nabla$ \\
4 & $0 \cdot 00020$ & $0 \cdot 012$ & $2 \cdot 5$ & $0 \cdot 050$ & $\mathrm{O}$ \\
5 & $0 \cdot 00020$ & $0 \cdot 011$ & $2 \cdot 1$ & $0 \cdot 060$ & $\Delta$ \\
6 & $0 \cdot 00020$ & $0 \cdot 010$ & $2 \cdot 0$ & $0 \cdot 062$ & $\square$ \\
\hline
\end{tabular}

tions are set to $\Delta t=0.01, \Delta t_{\mathrm{p}}=0.0001$ and $\Delta t_{\mathrm{C}}=0.000002$, where time is non-dimensionalized by $L / U$ as defined in the Computational method section. Extensive tests were conducted to ensure that the results are insensitive to the numerical parameters used to discretize the particle transport, including variation of the time step from $\Delta t$, the number of grid points used to interpolate the fluid velocity onto the particle centroids, the bed depth and the computational domain size (Yergey, 2008). These tests indicated that the particle velocity field does not change significantly with variation of these parameters by a modest fraction (e.g. a factor of two) about the values used for the current computations.

\section{COMPUTATIONAL RESULTS}

To evaluate the effects of the penetration depth on the onset of particle motion, the mean streamwise particle velocity $\overline{V_{\mathrm{t},+}}$ of the particles 


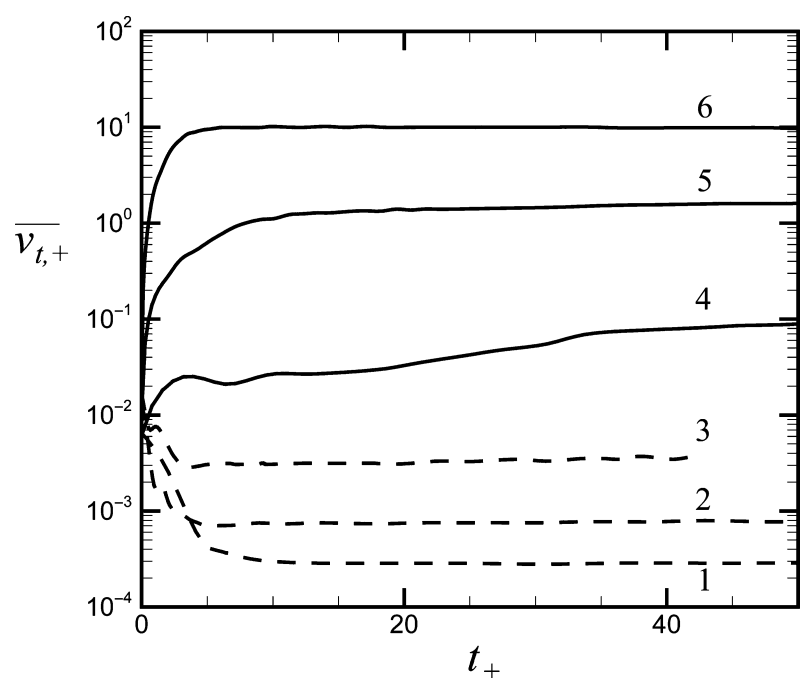

Fig. 3. Mean streamwise velocity of particles on the bed surface for Case 5 with dimensionless penetration depths $P_{+}=0.36,0.71,1.4,3.6,7 \cdot 1$ and $14 \cdot 2$, corresponding to lines numbered 1,2, 3, 4, 5 and 6 in the figure, respectively. Dashed lines are for cases for which the particle velocity decreases from its initial value and solid lines are for cases for which the particle velocity increases from its initial value.

at the top of the bed is computed by averaging the velocity of all particles having centroids within two particle diameters of the bed surface. A similar averaging process is performed for multiple sections within the bed from the bottom of the domain up through the highest particle location. Figure 3 shows the computed values of $\overline{V_{\mathrm{t},+}}$ for Case 5 with the six values of penetration depth considered. There is a small initial value of velocity due to residual vibrations of the particles following settling. For all values of penetration depth except $P_{+}=3.6$ (the curve numbered ' 4 ' in the plot), the particle velocity asymptotes to an approximately steady value, which might be either smaller or larger than the initial velocity. For the case with $P_{+}=3 \cdot 6$, the particle velocity continues to increase throughout the duration of the computation, although it appears to be starting to level out towards the end of the computation. Noting that the velocity scale in this plot is logarithmic, it is apparent that this measure of particle velocity is highly sensitive to the value selected for the penetration depth. In general, the streamwise particle velocity increases as the penetration depth increases. For low values of penetration depth, the mean particle velocity decreases rapidly after the flow is initiated, leading to an asymptotic value for particle velocity that is very small. A value of penetration depth exists in the interval

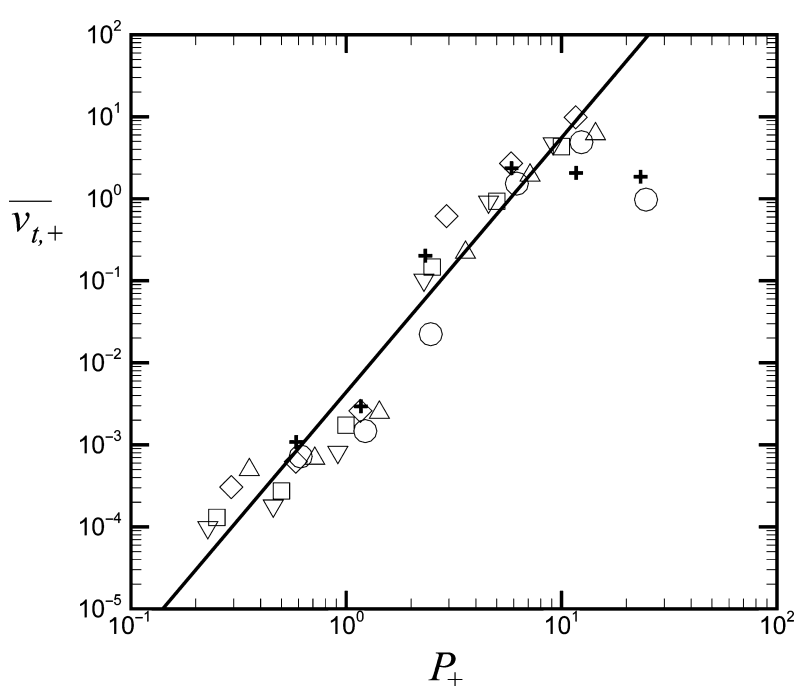

Fig. 4. Steady-state value of the mean streamwise velocity of particles on the bed surface as a function of penetration depth $P_{+}$for each of the six cases examined. Symbols correspond to the different cases as indicated in Table 1, and the curve is a best fit power law with slope approximately equal to 3 .

$1.4<P_{+}<3.6$ at which the trend in particle velocity changes from decreasing with time to increasing with time relative to its initial value. Only for $P_{+}$greater than this value are the particles, on average, accelerated by the fluid flow. Further increase in $P_{+}$causes additional acceleration, resulting in additional increase in particle streamwise velocity.

The steady-state values of the mean streamwise velocity at the top surface for all cases examined are plotted as a function of penetration depth in Fig. 4. Data from the different cases examined are observed to collapse onto a single curve, which is approximately fit for $P_{+}<10$ by a power law of the form $\overline{V_{\mathrm{t},+}}=C P_{+}^{3}$. For $P_{+}>10$, the data appear to deviate from the power-law fit, but the number of data points in this range is small so the precise trend for large $P_{+}$is not clear. As noted previously in this paper and by numerous other investigators, the determination of an incipient motion condition is somewhat arbitrary, depending on the experimental set-up and preciseness of the measurement instrumentation. For very low velocities, issues such as particle roughness and shape can also have a major effect on the particle motion. Figure 4 indicates the high degree of sensitivity that the incipient motion has to penetration depth. For a given symbol in the figure, all parameter values are held constant except the penetration depth, and yet the predicted particle velocity varies by nearly five orders of magnitude. 
The experimental observations of Gyr \& Schmid (1997) indicate a dimensionless slip velocity $u_{0}+$ of about 3.6 at the incipient motion condition in experiments with natural sediment having $d_{+} \cong 2 \cdot 2$ and $u_{*} \cong 1 \cdot 1 \mathrm{~cm} \mathrm{~s}^{-1}$. Recalling the result $P_{+}=u_{0+}$ from the Theory for velocity profile within the bed section, the Gyr \& Schmid (1997) slip value corresponds to $P_{+} \cong 3 \cdot 6$, which from Fig. 4 suggests predicted onset of visible particle motion at $\overline{v_{\mathrm{t},+}} \cong 0 \cdot 25$. For comparison, the mean fluid velocity at the top of the viscous sub-layer within the flat-plate turbulent boundary layer is about $u_{+} \cong 13$ (White, 2006).

Another method of evaluating the numerical results is by reviewing the particle velocities within the bed, where the particle velocity is averaged not just for particles within the top layer, but for multiple slices in the vertical direction. Figure 5 shows examples of the particle velocity profiles within the bed for a penetration depth of $P_{+}=3 \cdot 6$, coinciding with the observed slip velocity from Gyr \& Schmid (1997). Because the different cases have different total bed depths and slip velocities for the onset of particle motion, the curves are normalized such that the height within the bed is divided by the total bed depth (where in wall units $H_{+} \equiv H u * / v$ ) and the mean particle velocity at the given height is divided by the slip-velocity at the bed surface. The velocity is plotted on a logarithmic scale, such that an exponential velocity profile, such as

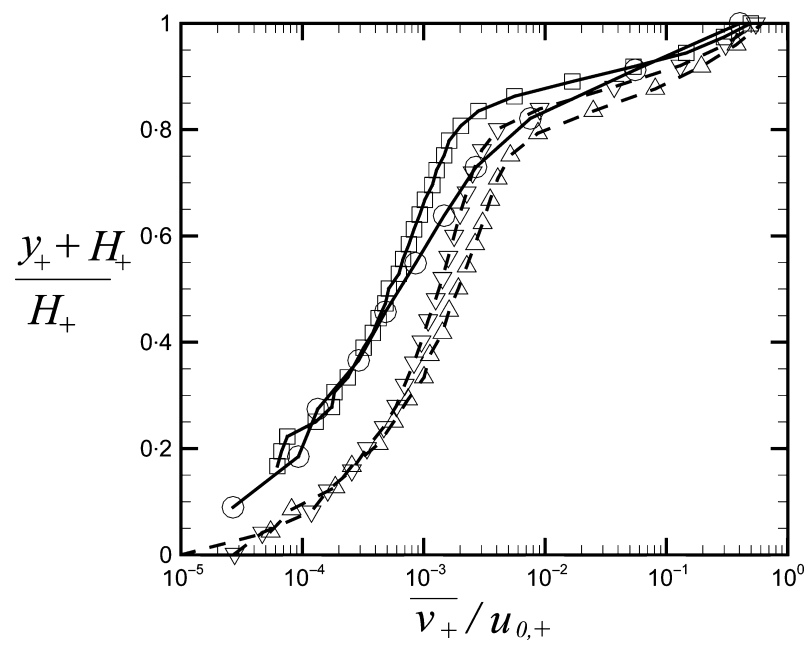

Fig. 5. Normalized mean steady-state streamwise particle velocity profiles for four different cases (using symbols defined in Table 1) for penetration depth $P_{+}=3 \cdot 6$. The non-dimensional vertical position $y_{+}$is normalized by the associated non-dimensional particle bed depth, and the non-dimensional mean streamwise particle velocity $\overline{V_{+}}$is normalized by the associated non-dimensional slip velocity $u_{0,+}$. obtained for the fluid in Eq. 8, would appear as a straight line in the plot. The plots are approximately linear in the upper $20 \%$ or so of the bed with a rapid decrease in particle velocity, followed by an abrupt change in slope as the velocity decays more slowly through the remaining part of the bed. Of course, these observations for particle velocity decay within the bed are for spheres with no rolling resistance. For natural sediment, the effects of non-spherical particle shape, roughness and rolling resistance may act to suppress particle motion within much of the bed.

Figure 6 shows the fluid and particle velocity profiles for Case 5 for two values of the penetration depth, $P_{+}=1.4$ and $P_{+}=3.6$. For the case with $P_{+}=1.4$ in Fig. $6 \mathrm{~A}$, there is little observable
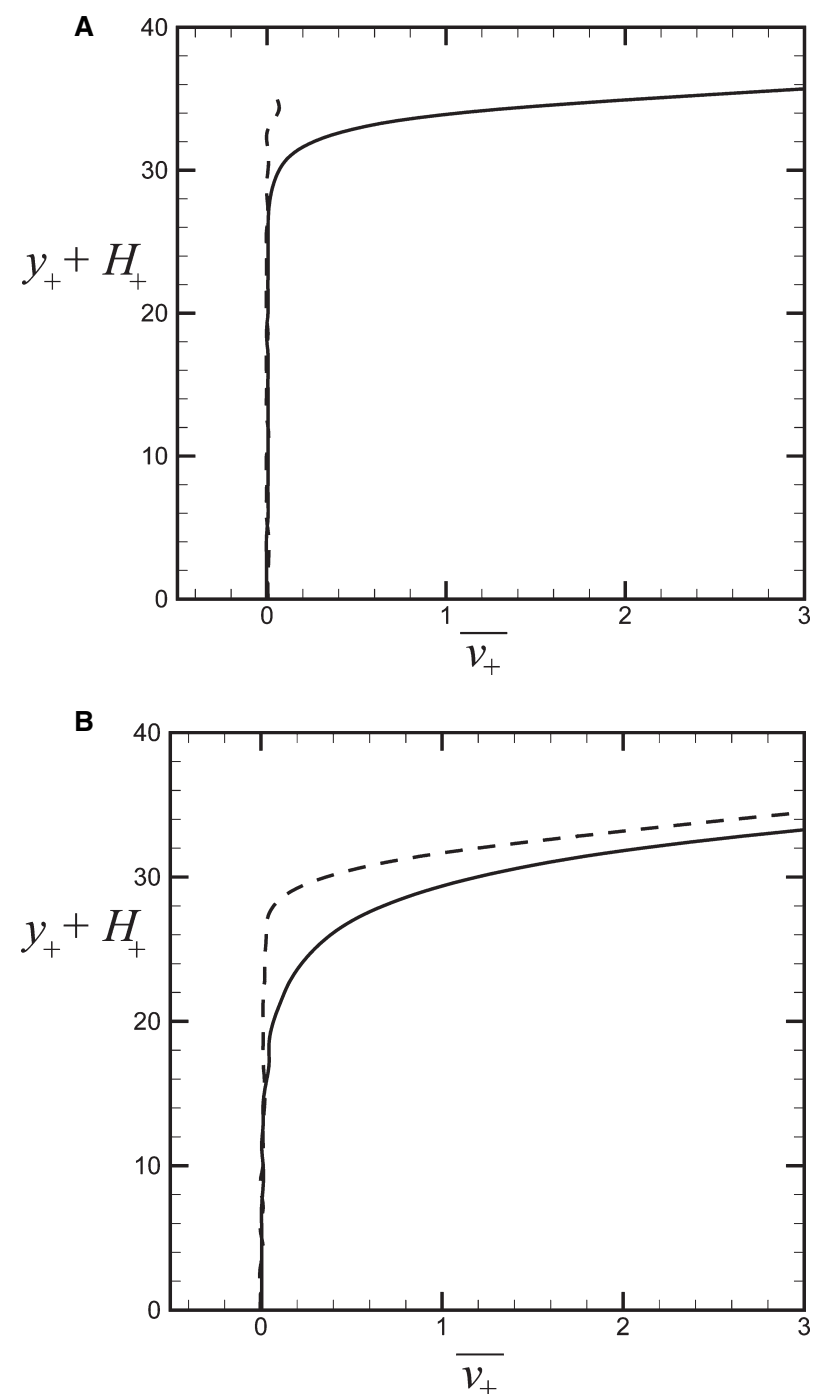

Fig. 6. Mean steady-state streamwise velocity profile particles (dashed line) and fluid (solid line) for Case 5 with penetration depths (A) $P_{+}=1.4$ and (B) $P_{+}=3.6$. 
particle motion. For the case with $P_{+}=3 \cdot 6$ in Fig. 6B, the particles move in the direction of the fluid flow, but consistently lag the fluid velocity. The particle velocity appears to nearly vanish (in the scale used for this figure) below a height of $y_{+}+H_{+}=28$, despite the fact that there is significant fluid velocity at this height.

\section{DISCUSSION AND CONCLUSIONS}

The study demonstrates that incipient motion of particles at the bed surface is sensitive to the fluid velocity field within the bed (i.e. the penetration depth) and, hence, to the constitutive makeup of the bed material. The discrete element method (DEM) presented in the current study yields reasonable predictions for particle incipient motion provided that the penetration depth of the fluid velocity profile within the bed is set to a value consistent with experimentally observed values of the slip velocity at the bed surface. Once particles begin moving in the computations, it is observed that the particle velocity within the bed substantially lags the fluid velocity at the same location. While the particle velocity in the upper part of the bed exhibits an exponential decay with depth, the rate of this exponential decay changes abruptly at a certain depth which, in the cases examined here, occurs at a depth below the surface of about $20 \%$ of the total bed depth.

It is clear from this study that it is important to include the effect of fluid flow within the bed when developing models for sediment transport using the DEM, even though the fluid velocity within the bed is generally very small. Although in the current study the fluid flow was estimated using a flat bed with stationary particles, in general particle entrainment into the flow, bedload transport and formation of bedforms will all have a significant effect on the near-bed fluid flow. Future DEM models need to be extended and coupled to the fluid velocity computation to accurately model the particle effect on the fluid flow in this near-bed region. This study illustrates a need for more detailed research and modelling efforts on a number of topics in order to advance a new generation of micro-scale sediment transport models. In particular, there is a need for more accurate models of lift force on particles that lie in regions of strong concentration gradient, such as at the top of the particle bed. Furthermore, there is a need for better models of particle effects on the fluid motion which can be used for entrained particles in the bedload layer and for cases with bedforms and mixtures of sediment of different sizes.

\section{NOMENCLATURE}

\section{Roman}

$B \quad$ Average conductance of porous medium

$C \quad$ Coefficient in power law shown in Fig. 4

d Particle diameter

D Fluid rate of deformation tensor

$e \quad$ Particle restitution coefficient

E Effective elastic modulus of two colliding particles

$E_{p} \quad$ Particle elastic modulus

$f^{p} \quad$ Friction factor for particle drag force

f Fluid body force due to particle-fluid interaction

$F_{\text {crit }} \quad$ Critical sliding resistance force $\left[=\mu_{f}\left|F_{n}\right|\right]$

$\mathbf{F}_{\mathrm{C}} \quad$ Particle collision force

$\mathbf{F}_{\mathrm{d}} \quad$ Drag force on particle

$\mathbf{F}_{\ell} \quad$ Lift force on particle

$\mathbf{F}_{\mathrm{m}} \quad$ Magnus force

$F_{\mathrm{n}} \quad$ Normal force between two colliding particles

$F_{\text {nd }} \quad$ Normal dissipative force

$F_{\text {ne }} \quad$ Normal elastic force

$F_{\mathrm{s}} \quad$ Sliding resistance force between two colliding particles

g Gravitational acceleration

$H$ Bed depth

$k_{N} \quad$ Particle spring coefficient in Hertz expression

$K \quad$ Particle elastic resistance coefficient $\left[\equiv k_{N} / \delta_{N}^{1 / 2}\right]$

$L \quad$ Fluid length-scale $\left[=\left(v / u_{*}\right) \times 10^{2}\right]$

$m$ Particle mass

$\mathbf{M}_{\mathrm{C}} \quad$ Particle collision torque

$M_{\mathrm{F}} \quad$ Particle viscous torque on particle

$M_{\mathrm{t}} \quad$ Twisting resistance torque

n Normal vector for collision between two particles

$p \quad$ Fluid pressure field

$P \quad$ Fluid penetration depth $[\equiv \sqrt{B}]$

$r_{i} \quad$ Radius of particle $i$

$R \quad$ Effective radius of two colliding particles

$\operatorname{Re}_{P} \quad$ Particle Reynolds number $[\equiv|\mathbf{v}-\mathbf{u}| d / v]$

$S \quad$ Fluid shear rate

St Particle Stokes number $\left[\equiv \rho_{p} d^{2} U / 18 \mu \mathrm{L}\right]$

$t \quad$ Time

$\mathbf{t}_{\mathrm{S}} \quad$ Unit vector in the direction of sliding

$\Delta t \quad$ Fluid time step $[=O(L / U)]$

$\Delta t_{\mathrm{p}} \quad$ Particle time step [=O(d/U)]

$\Delta t_{\mathrm{c}} \quad$ Collision time step $\left[=O\left(d\left(\rho_{\mathrm{p}}^{2} / E_{\mathrm{p}}^{2} U\right)^{1 / 5}\right)\right]$ 
u Spatially averaged fluid velocity vector

$u_{0} \quad$ Slip velocity at bed surface

$u_{*} \quad$ Friction velocity $\left[\equiv\left(\tau_{w} / \rho_{f}\right)^{1 / 2}\right]$

$U \quad$ Fluid velocity scale $\left[=u_{*}\right]$

v Particle velocity field

$\mathbf{v}_{\mathrm{R}} \quad$ Relative velocity between two particles $\left[=\mathbf{v}_{i}-\mathbf{v}_{j}\right]$

$\overline{v_{t,+}} \quad$ Average dimensionless particle velocity within bed top layer (of width $2 d$ )

$x \quad$ Co-ordinate tangent to bed surface in direction of mean flow

$\mathbf{x}_{i} \quad$ Centroid of particle $i$

y Co-ordinate normal to bed surface

z Co-ordinate tangent to bed surface in crossstream direction to mean flow

\section{Greek}

$\alpha$ Coefficient in lift force equation $[\equiv \omega d / 2|\mathbf{v}-\mathbf{u}|]$

$\bar{\alpha} \quad$ Coefficient in dissipation term

$\chi \quad$ Density ratio $\left[\equiv \rho_{\mathrm{f}} / \rho_{\mathrm{p}}\right]$

$\delta_{N} \quad$ Particle overlap

$\varepsilon \quad$ Void fraction $[\equiv 1-\phi]$

$\phi \quad$ Particle concentration field

$\eta_{N} \quad$ Dissipation coefficient $\left[=\alpha\left(m k_{N}\right)^{1 / 2}\right]$

$\mu \quad$ Fluid viscosity

$\mu_{\mathrm{f}} \quad$ Particle friction coefficient

$v \quad$ Fluid kinematic viscosity $\left[\equiv \mu / \rho_{f}\right]$

$\theta_{\text {crit }} \quad$ Shields parameter $\left[=\tau_{\text {crit }} / d g\left(\rho_{\mathrm{p}}-\rho_{\mathrm{f}}\right)\right]$

$\rho_{\mathrm{f}} \quad$ Fluid density

$\rho_{\mathrm{p}} \quad$ Particle density

$\tau_{\text {crit }}$ Critical wall stress for incipient motion (as defined by Shields)

$\tau_{\mathrm{w}} \quad$ Wall shear stress

$\omega \quad$ Vorticity magnitude $[\equiv|\omega|]$

$\boldsymbol{\omega} \quad$ Averaged fluid vorticity vector $[\equiv \nabla \times \mathbf{u}]$

$\Omega \quad$ Particle rotation rate

\section{Subscripts}

$+\quad$ Non-dimensionalized by turbulent wall units [Eq. 8]

\section{REFERENCES}

Acrivos, A. and Chang, E. (1986) A model for estimating transport quantities in two-phase materials. Phys. Fluids, 29, 3-4.

Bear, J. (1988) Dynamics of Fluids in Porous Media. Dover Publications, New York.

Beavers, G.S. and Joseph, D.D. (1967) Boundary conditions at a naturally permeable wall. J. Fluid Mech., 30, 197-207.

Brinkman, H.C. (1947) A calculation of the viscous force exerted by a flowing fluid on a dense swarm of particles. Appl. Sci. Res., 1, 27-34.
Chang, Y. and Scotti, A. (2003) Entrainment and suspension of sediments into a turbulent flow over ripples. J. Turbulence, 4, 019-1-019-22.

Chen, X. and Chiew, Y.-M. (2004) Velocity distribution of turbulent open-channel flow with bed suction. J. Hydraul. Eng., 130, 140-148.

Crowe, C., Sommerfeld, M. and Tsuji, Y. (1998) Multiphase Flows with Droplets and Particles. CRC Press, Boca Raton, FL, pp. 95-99.

Cundall, P.A. and Strack, O.D.L. (1979) A discrete numerical model for granular assembles. Geotechnique, 29, 47-65.

Darcy, H. (1856) Les fontaines publiques de la ville de Dijon. Dalmont, Paris.

Dey, S. and Papanicolaou, A. (2008) Sediment threshold under stream flow: a state-of-the-art review. KSCE J. Civil Eng. 12, 45-60.

Di Felice, R. (1994) The voidage function for fluid-particle interaction systems. Int. J. Multiphase Flow, 20, 153-159.

Drake, T.G. and Calantoni, J. (2001) Discrete particle model for sheet flow sediment transport in the nearshore. J. Geophys. Res., 106(C9), 19859-19868.

Egiazaroff, I.V. (1950) Coefficient $f$ de la force d'entrainment critique des materiaux par charriage. Proceedings of Academy of Sciences, Armenian Soviet Socialist Republic, Translation Number 499, Electricité de France Service des Etudes et Récherches Hydrauliques, Paris, France.

Fenton, J.D. and Abbott, J.E. (1977) Initial movement of grains on a stream bed: the effect of relative protrusion. Proc. $R$. Soc. London A, 352, 523-537.

Foster, D.L., Bowen, A.J., Holman, R.A. and Natoo, P. (2006) Field evidence of pressure gradient induced incipient motion. J. Geophys. Res., 111, C05004.1-C05004.8.

Fries, J.S. (2007) Predicting interfacial diffusion coefficients for fluxes across the sediment-water interface. J. Hydraul. Eng., 133, 267-272.

Fries, J.S. and Trowbridge, J.H. (2003) Flume observations of enhanced fine-particle deposition to permeable sediment beds. Limnol. Oceanogr., 48, 802-812.

Gyr, A. and Schmid, A. (1997) Turbulent flows over smooth erodible sand beds in flumes. J. Hydraul. Res., 35, 525-544.

Hammond, F.D.C., Heathershaw, A.D. and Langhorne, D.N. (1984) A comparison between Shield's threshold criterion and the movement of loosely packed gravel in a tidal channel. Sedimentology, 31, 51-62.

Hertz, H. (1882) Über die Berührung fester elastische Körper. $J$. reine und angewandte Mathematik, 92, 156-171.

James, D. and Davis, A. (2001) Flow at the interface of a model fibrous porous medium. J. Fluid Mech., 426, 47-72.

Joseph, G., Zenit, R., Hunt, M. and Rosenwinkel, A. (2001) Particle-wall collisions in a viscous fluid. J. Fluid Mech., 433, 329-346.

Larson, R.E. and Higdon, J.J.L. (1986) Microscale flow near the surface of two-dimensional porous media. Part 1. Axial flow. J. Fluid Mech., 166, 449-472.

Larson, R.E. and Higdon, J.J.L. (1987) Microscale flow near the surface of two-dimensional porous media. Part 2. Transverse flow. J. Fluid Mech., 178, 110-136.

Ling, C. (1995) Criteria for incipient motion of spherical sediment particles. J. Hydraul. Eng., 121, 472-478.

Marshall, J.S. (2006) Effect of shear-induced migration on the expulsion of heavy particles from a vortex core. Phys. Fluids, 18, 113301-1-113301-12.

Marshall, J.S. (2007) Particle aggregation and capture by walls in a particulate aerosol channel flow. J. Aerosol Sci., 38, 333-351. 
Marshall, J.S. (2009) Discrete-element modeling of particulate aerosol flows. J. Comput. Phys., 228, 1541-1561.

McEwan, I. and Heald, J. (2001) Discrete particle modeling of entrainment from flat uniformly sized sediment beds. J. Hydraul. Eng., 127, 588-597.

Niño, Y., Lopez, F. and Garcia, M. (2003) Threshold for particle entrainment into suspension. Sedimentology, 50, 247-263.

Pender, G., Shvidchenko, A.B. and Chegini, A. (2007) Supplementary data confirming the relationship between critical Shields stress, grain size and bed slope. Earth Surf. Proc. Land., 32, 1605-1610.

Phillips, M. (1980) A force balance model for particle entrainment into a fluid stream. J. Phys. D: Appl. Phys., 13, 221-233.

Pokrajac, D., Manes, C. and McEwan, I. (2007) Peculiar mean velocity profiles within a porous bed of an open channel. Phys. Fluids, 19, 098109-1-098109-4.

Saffman, P.G. (1965) The lift on a small sphere in a slow shear flow. J. Fluid Mech., 22, 385-400.

Saffman, P.G. (1968) Corrigendum to 'The lift force on a small sphere in a slow shear flow'. J. Fluid Mech., 31, 624.

Sahraoui, M. and Kaviany, M. (1992) Slip and no-slip velocity boundary conditions at interface of porous, plain media. International Journal of Heat and Mass Transfer, 35, 927943.

Sangani, A.S. and Behl, S. (1989) The planar singular solutions of Stokes and Laplace equations and their applications to transport processes near porous surfaces. Phys. Fluids A, 1, 21-37.

Schmeeckle, M. and Nelson, J. (2003) Direct numerical simulation of bedload transport using a local dynamic boundary condition. Sedimentology, 50, 279-301.

Schmeeckle, M., Nelson, J., Pitlick, J. and Bennett, J. (2001) Interparticle collision of natural sediment grains in water. Water Resour. Res., 37, 2377-2391.

Shields, A.F. (1936) Anwendung der Aehnlichkeitsmechanik und der Turbulenzforschung auf die Geschiebebewegung.
Mitteilungen der Preussischen Versuchsanstalt fur Wasserbau und Schiffbau, 26, 1-26 (Berlin).

Shvidchenko, A.B. and Pender, G. (2000) Flume study of the effect of relative depth on the incipient motion of course uniform sediments. Water Resour. Res., 36, 619-628.

Taylor, G.I. (1971) A model for the boundary condition of a porous material. Part 1. J. Fluid Mech., 49, 310-326.

Tison, L.J. (1953) Studies of the critical tractive force for the entrainment of bed materials. Proceedings of the Minnesota International Hydraulics Conference, Minneapolis, MN, pp. 21-35.

Tsuji, Y., Tanaka, T. and Ishida, T. (1992) Lagrangian numerical simulation of plug flow of cohesionless particles in a horizontal pipe. Powder Technol. 71, 239-250. Tsuji (1992)

Vanoni, V.A. (Ed) (1975), Sedimentation Engineering. American Society of Civil Engineers, New York, p. 94.

White, C.M. (1940) The equilibrium of grains on the bed of a stream. Proc. R. Soc. London A 174, 322-338.

White, F.M. (2006) Viscous Fluid Flow. McGraw-Hill Inc., New York, p. 430.

Yergey, B.A. (2008) Analysis of sediment entrainment; exponential decay for flow within the particle bed. MS Thesis, Bucknell University.

Zanke, U.C.E. (2003) On the influence of turbulence on the initiation of sediment motion. Int. J. Sed. Res., 18, 17-31.

Zedler, E.A. and Street, R.L. (2001) Large-eddy simulation of sediment transport: current over ripples. J. Hydraulic Eng. 127, 444-452.

Zhao, Y. and Marshall, J.S. (2008) Spin coating of a colloidal suspension. Phys. Fluids, 20, 043302-1-043302-15.

Zhao, X.-L., Li, S.-Q., Liu, G.-Q., Yao, Q. and Marshall, J.S. (2008) DEM simulation of the particle dynamics in twodimensional spouted beds. Powder Technol., 184, 205-213.

Manuscript received 26 January 2009; revision accepted 5 June 2009 